\title{
Effect on the liver cancer cell invasion ability by studying the associations between autophagy and TRAP1 expression
}

\author{
YI SUN $^{1}$, HONGLING ZOU ${ }^{2}$, LIU YANG ${ }^{1}$, MENGTING ZHOU $^{1}$, XIAOLING SHI ${ }^{1}$, \\ YARUI YANG ${ }^{3}$, WENJUN CHEN ${ }^{1}$, YINGQI ZHAO ${ }^{1}$, JIE MO $^{1}$ and YUANMING LU ${ }^{1}$ \\ ${ }^{1}$ Department of Toxicology, Guilin Medical University; ${ }^{2}$ Department of Oncology, Second Affiliated Hospital of \\ Guilin Medical College, Guilin, Guangxi 541004; ${ }^{3}$ Laboratory of Molecular Biology, Shenzhen \\ Nanshan Center for Chronic Disease Control, Shenzhen, Guangdong 518054, P.R. China
}

Received November 9, 2017; Accepted May 3, 2018

DOI: $10.3892 / \mathrm{ol} .2018 .8774$

\begin{abstract}
Liver cancer is one of the leading causes of cancer associated mortality, particularly in eastern Asia. Autophagy serves an important role in carcinogenesis. Previous studies have reported that TRAP1 is a novel and efficient therapeutic target in various tumors. However, the associations between autophagy and TRAP1 is not clear. In the present study, autophagy activity and TRAP1 expression were examined in 4 different liver cancer cell lines (HepG2, Hep3B2.1-7, Sk-hep1 and HepG2.2.15) with or without rapamycin induction. The cell autophagy level was validated by monodansylcadaverine fluorescent staining, and the expression levels of Beclin1 and light chain (LC)-3-II/LC3-I. The mRNA and protein expression levels of tumor necrosis factor receptor-associated protein-1 (TRAP-1), Beclin1 and LC3-II/LC3-I were measured by reverse transcription-quantitative polymerase chain reaction, Protein Simple Western and western blot analysis. HepG2 cells, with medium invasive ability, exerted the highest basal level of autophagy and TRAP1 expression. In addition, hepatitis B (HBV) infection in HepG2 cells inhibited autophagy activity and TRAP1 expression. Rapamycin treatment also significantly enhanced autophagy in the 4 liver cancer cell lines and increased TRAP1 expression in HepG2, Hep3B2.1-7 and Sk-hep1 cells. Thus, the cell invasive ability, HBV infection and autophagy induction had different effects on TRAP1 expression, and TRAP1 may be associated with autophagy in liver cancer.
\end{abstract}

Correspondence to: Professor Yuanming Lu, Department of Toxicology, Guilin Medical University, 1 Zhiyuan Road, Guilin, Guangxi 541004, P.R. China

E-mail: bobojpn2002@163.com

Key words: tumor necrosis factor receptor-associated protein-1, hepatitis B, liver cancer, autophagy, invasive ability

\section{Introduction}

Primary liver cancer (including hepatocellular carcinoma and intrahepatic cholangiocarcinoma) is the second cause of cancer related death and one of the cancers with still increasing incidence rate $(1,2)$. Hepatocellular carcinoma, a major primary liver cancer pathological type, shows very low 5-year survival rate $(<15 \%)$ owing to its late diagnosis and compromised underlying liver function $(3,4)$. Although surgical resection or transplantation helps to improve the survival rate of patients, there is still no effective treatment for advanced patients who are ineligible for surgery, resulting in only a median overall survival of 6.6 months for these patients $(5,6)$. Therefore, it is imperative to explore effective and safe prognostic biomarkers for early diagnosis and therapeutic targets to improve treatment strategies.

Autophagy is a dynamic degradation process for delivering dysfunctional cellular components or foreign invaders to lysosome to be digested by lysosomal hydrolase (7-9). Autophagy-related gene (ATG) products play important roles in autophagy. Autophagosome formation is mediated by two ubiquitin-like conjuction systems composed of ATG proteins, which culminate in conjugation of ATG12 to ATG5 and conversion of a soluble form of microtubule-associated protein 1 light chain 3 (LC3-I) to phosphatidylethanolamine-conjugated membrane-bound form (LC3-II) $(10,11)$. Beclin1 is a component of the class III phosphoinositide 3-kinase complex and also plays an essential role in autophagy regulation. A considerable body of studies have shown that autophagy plays a dual-side role in cancer pathogenesis. In the initiation of tumor, autophagy may inhibit tumor formation by degradation of damaged organelles or proteins (12). After the formation of tumor, however, the tumor can utilize the autophagy as a survival mechanism to counter metabolic stress (13).

Additionally, recent evidence has demonstrated that autophagy is linked to increased tumor cell invasion because tumor cell invasion requires the production of pro-migratory cytokines mediated by autophagy $(14,15)$. How autophagy activity influence cancer metastasis remains to be determined.

It is known that heat shock protein 90 (HSP90) chaperones are pivotal regulators of proteostasis in the mitochondria of selective tumor cells. TNF receptor-associated 
protein-1 (TRAP1), as a mitochondrial molecular chaperone of the Hsp90 family, has attracted attention because of its high expression in various tumors but low expression in normal tissues $(16,17)$. HSP90 chaperones can work as an upstream connector between the control of protein folding and adaptive mechanisms of autophagy and cancer metabolism. Some recent studies have shown that the TRAP1 expression level and cellular mitochondria autophagy are closely related (18-21). However, the relationship between TRAP1 expression and autophagy in liver cancer is still unknown.

In the present study, we aim to explore the effect of rapamycin, invasive ability and hepatitis $\mathrm{B}(\mathrm{HBV})$ infection on the level of autophagy and TRAP1 expression in four different liver cancer cell lines and the relationship between autophagy and TRAP1 in liver cancer.

\section{Materials and methods}

Reagents. The following reagents were used: EMDM (Gibco; Thermo Fisher Scientific, Inc., Waltham, MA, USA); FBS (Gemini Bio Products, West Sacramento, CA, USA); TRIzol (Ambion; Thermo Fisher Scientific, Inc.); MDC (Tiangen Biotech Co., Ltd., Beijing, China); WIP organization cell lysis solution (Boaoseng, Beijing, China); RNase Away (Ed lai, Beijing, China); BCA protein concentration determination kit (Blue skies, China); SDS-PA (Nanjing KeyGen Biotech. Co. Ltd., Nanjing, China); Reverse transcription kit (Lisu, Shanghai, China); Primers (Hongxun, Suzhou, China); High sensitive chemiluminescence detection kit (Qihaifutan, Shanghai, China); TRAP1 Rabbit mAb (Cell Signaling Technology, Inc., Danvers, MA, USA); Beclin1 Rabbit mAb (Cell Signaling Technology, Inc.); LC3 Rabbit mAb (Cell Signaling Technology, Inc.); Anti-rabbit IgG, HRP-linked Antibody (Isotype: Goat; Cell Signaling Technology, Inc.); Mouse anti $\beta$-actin mAb (Zhongshanjinqiao, China); Anti- $\beta$-actin Mouse Monoclonal Antibody (Isotype: Goat; Zhongshanjinqiao, China); and Protein Simple (San Jose, CA, USA).

Cell lines and culture. Four liver cancer cell lines (Hep3B2.1-7, Sk-hep1, HepG2 and HepG2.2.15 cell lines) were obtained from the Chinese Academy of Sciences Library. All cell lines were cultured in EMDM medium containing 10\% FBS and were maintained at $37^{\circ} \mathrm{C}$ in $95 \%$ air and $5 \% \mathrm{CO}_{2}$.

RNA isolation and reverse transcription. Total RNA was isolated from four liver cancer cell lines with TRIzol according to manufacturer's instructions. After the RNA concentration was measured, the integrity of the isolated RNA was analyzed (NanoDrop 2000; Thermo Fisher Scientific, Inc.), $3 \mu \mathrm{g}$ of RNA was reverse transcribed into cDNA according to the manufacturer's protocol.

Reverse transcription-quantitative polymerase chain reaction $(R T-q P C R)$. RT-qPCR was performed using SYBR-Green Supermix. The reaction conditions were as follows: $95^{\circ} \mathrm{C}$ for $10 \mathrm{sec}$ and $60^{\circ} \mathrm{C}$ for $32 \mathrm{sec}, 40$ cycles. Target genes were normalized to $\beta$-actin and were quantified using the comparative $2^{-\Delta \Delta \mathrm{Cq}}$ method. TRAP1 expression levels were measured in triplicate, and the average was calculated. $\beta$-actin was applied as an internal control. The primers for $\beta$-actin
(96 bp) were 5'-GATCAGCAGCAGAGTATG-3' (F) and 5'-AGAGTGTACGCACTA-3' (R). The primers for TRAP-1 (112 bp) were 5'-CAGGGTTCCACTTCCAAACA-3' (F) and 5'-TGGAGATCAGCTCCCGTATAA-3' (R).

Rapamycin-induced cell autophagy. A total of $3 \times 10^{5} / \mathrm{ml}$ cells in the logarithmic growth phase were inoculated in a round-bottom 6 -well plate. The cells were exposed to rapamycin at $0,5,10$, and $15 \mu \mathrm{mol} / \mathrm{l}$ for $10,15,20,25$ and $30 \mathrm{~h}$. Next, the cells were washed three times with PBS, dyed for 30 min (MDC) in the dark, and washed three times with $1 x$ wash buffer. Finally, infiltrating cells were obtained with collection buffer. The cells were observed and photographed by using a fluorescence microscope (Eclipse Ti-U; Nikon Corporation, Tokyo, Japan). We found that activation of autophagy was highest when the cells were induced by rapamycin for $20 \mathrm{~h}$. Thus, we treated the cells with rapamycin for $20 \mathrm{~h}$ in the present study.

Western blot analysis. Thirty micrograms of protein samples from each case was separated with $10 \%$ sodium dodecyl sulfate-polyacrylamide gel electrophoresis and were subsequently transferred to polyvinylidene fluoride membranes. After transfer, the membranes were incubated with blocking buffer of 5\% non-fat dry milk in Tris-buffered saline containing Tween-20 at room temperature for $1 \mathrm{~h}$. The membranes were incubated with primary antibodies (1:1,000 dilution) for $20 \mathrm{~h}$ at $4^{\circ} \mathrm{C}$. After washing, secondary antibodies (1:5,000 dilution) were added at room temperature for $1 \mathrm{~h}$. Finally, ECL development and fixing were considered.

Protein Simple western analysis. Protein levels were quantified by using WES ${ }^{\mathrm{TM}}$ (Protein Simple), an automated capillary-based size sorting system (22). Protein samples and 5x Fluorescent master were mixed in a micro-centrifuge tube (final concentration, $0.2 \mathrm{mg} / \mathrm{ml}$ ); then, the samples were denatured, and a biotinylated ladder was used $\left(95^{\circ} \mathrm{C}, 5 \mathrm{~min}\right)$. The primary antibody was diluted 1:50 in Antibody Diluent II before use. The secondary antibody was used without dilution (Protein Simple). Next, $150 \mu$ l of Luminol-S and $150 \mu 1$ of peroxide were combined in a micro-centrifuge tube. The samples, wash buffer, primary antibodies, secondary antibodies, blocking reagent, and chemiluminescent substrate were dispensed into designated wells in the provided microplate. Following plate loading, separation and immunodetection were performed automatically using default settings. The incubation time of the primary antibody was $60 \mathrm{~min}$ and that of the secondary antibodies was $30 \mathrm{~min}$. The data were analyzed using inbuilt Compass software (Protein Simple).

Cell growth curve. Overall, $1 \times 10^{4} / \mathrm{ml}$ cells in the logarithmic growth phase were inoculated in a round-bottomed 6-well plate. Starting on the second day, the cells with pancreatic enzyme digestion and count three holes every day, averaged, and counted for five consecutive days.

Statistical analysis. All P-values were two sided, and $\mathrm{P}<0.05$ was considered to indicate a statistically significant difference. The experimental data were expressed as the mean \pm standard deviation, and analysis of variance with an LSD t-test was used to assess the relationship between two groups. Statistical 

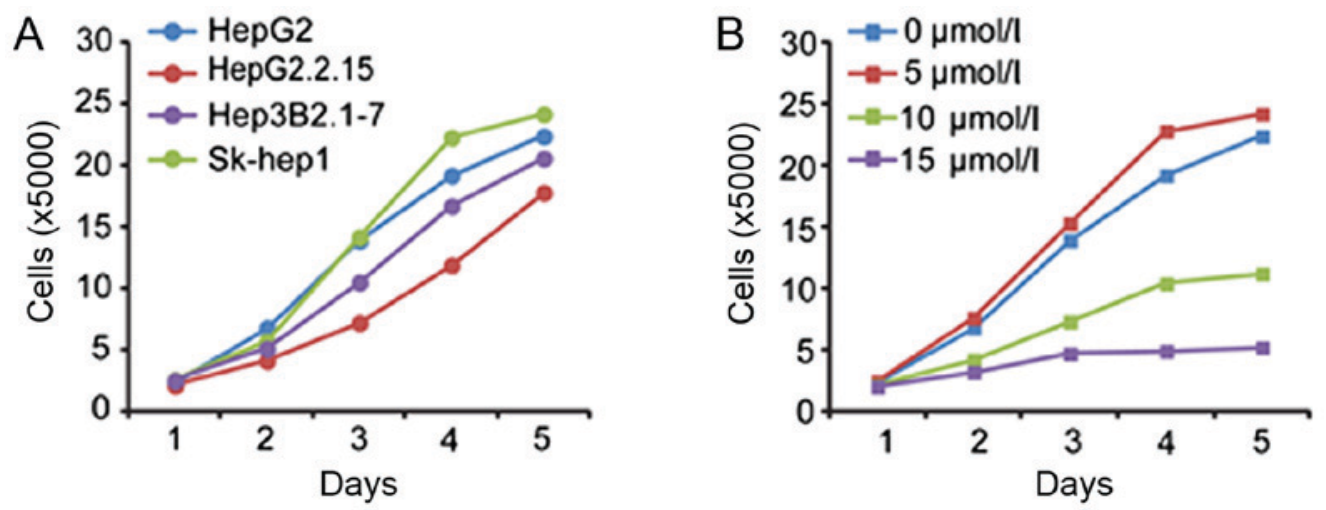

Figure 1. (A) Cell growth of HepG2, HepG2.2.15, Sk-hep1 and Hep3B2.1-7 cells was examined by cell counting over 5 days. (B) Growth curves of HepG2 cells following treatment with $0,5,10$ and $15 \mu \mathrm{mol} / 1$ rapamycin.
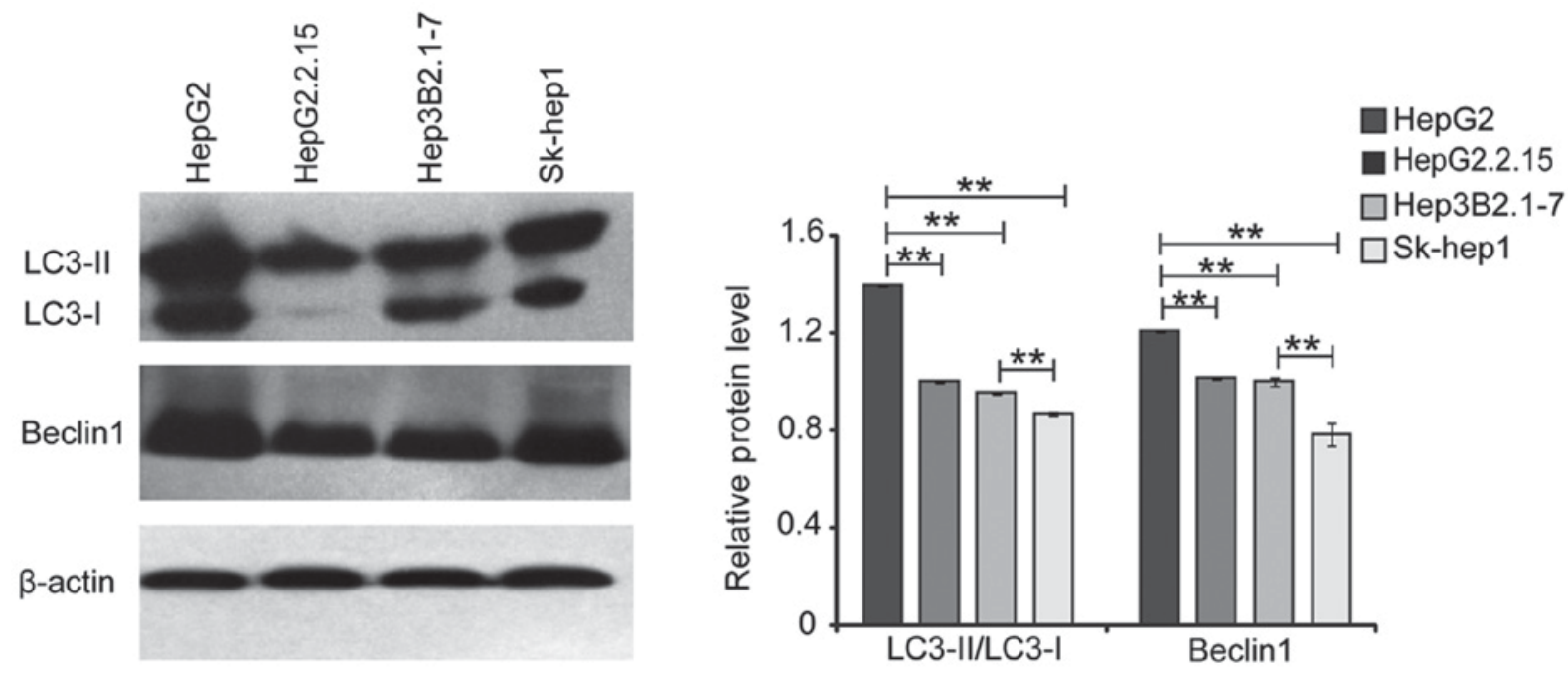

Figure 2. Protein levels of Beclin1 and LC3-II/LC3-I in 4 liver cancer cell lines were determined by western blot analysis. $\beta$-actin was used as an internal control. ${ }^{* *} \mathrm{P}<0.01$, as indicated. LC 3 , light chain 3 .

analyses were performed using the SPSS v20.0 software (SPSS, Inc., Chicago, IL, USA).

\section{Results}

Cell invasive ability, $H B V$ infection and rapamycin influenced cell growth. Herein, cell growth was examined in four liver cancer cell lines. Hep3B2.1-7, HepG2 and Sk-hep1 cells, which exhibit growing invasive ability, showed increasing cell proliferation ability, which indicated that cell invasive ability was positively related to cell growth. Additionally, the growth rate of HepG2.2.15 cells was slower when compared with HepG2 cells $(\mathrm{P}<0.05)$, suggesting that $\mathrm{HBV}$ infection inhibited the growth of cells (Fig. 1A).

We also examined the effect of rapamycin on the growth of HepG 2 cells at $0,5,10$ and $15 \mu \mathrm{mol} / 1$ for $20 \mathrm{~h}$. As a result, the growth rate of HepG2 cells was inhibited under the treatment of 10 and $15 \mu \mathrm{mol} / 1$ rapamycin $(\mathrm{P}<0.05)$. The results indicated that rapamycin exposure could inhibit the growth of HepG2 cells (Fig. 1B).

Basal level of autophagy was affected by cell invasive ability and HBV infection. The basal level of autophagy was examined in four liver cancer cell lines. Beclin1 and LC3-II/LC3-I are two specific autophagy-related markers and their protein level were detected by western blot. As shown in Fig. 2, the expression level of Beclin1 and LC3-II/LC3-I was changed in the same tendency among HepG2, Hep3B2.1-7, Sk-hep1 and HepG2.2.15 cells. HepG2 cell line, with medium invasive ability, showed the highest expression level of Beclin1 and LC3-II/LC3-I. However, the expression level of Beclin1 and LC3-II/LC3-I was lowest in the most invasive Sk-hep1 cell line. These findings suggested that the basal level of autophagy might be affected by cell invasive ability. Moreover, when compared to HepG2 cells, the protein level of Beclin1 and LC3-II/LC3-I were significantly decreased in HBV-infected HepG2.2.15 cells, indicating that the HBV infection might inhibit basal level of cell autophagy.

Rapamycin triggered autophagy in four liver cancer cell lines. Rapamycin is a mTOR-independent autophagy inducer. Four liver cancer cell lines were further treated with indicated concentration of rapamycin for $20 \mathrm{~h}$. As shown in Fig. 3A, compared with control group, the expression levels of Beclin1 and LC3-II/LC3-I were significantly increased in rapamycin-treated cells $(\mathrm{P}<0.05)$. In addition, the highest expression levels were 
A

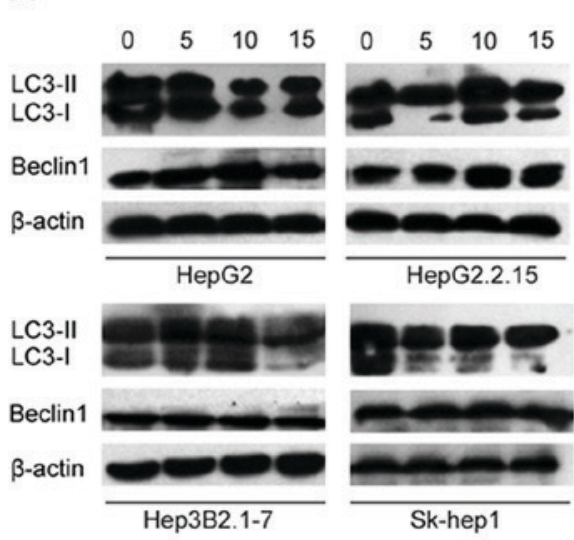

B

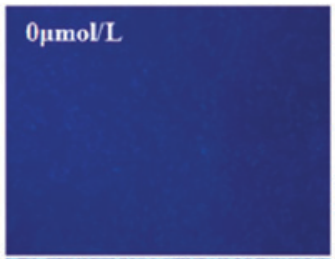

\section{$10 \mu \mathrm{molL}$}

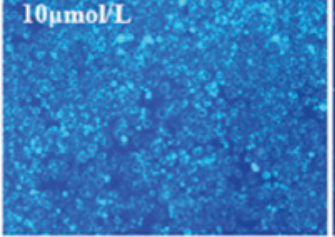

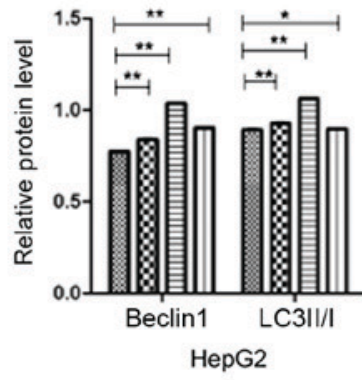

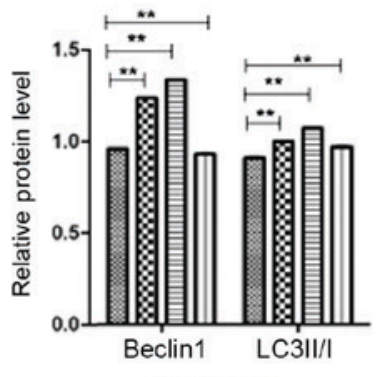

Hep3B2.1-7
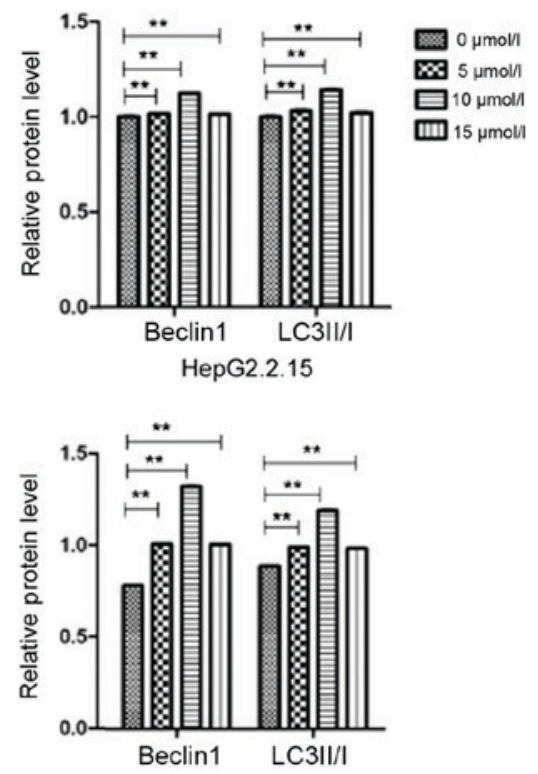

Sk-hep1
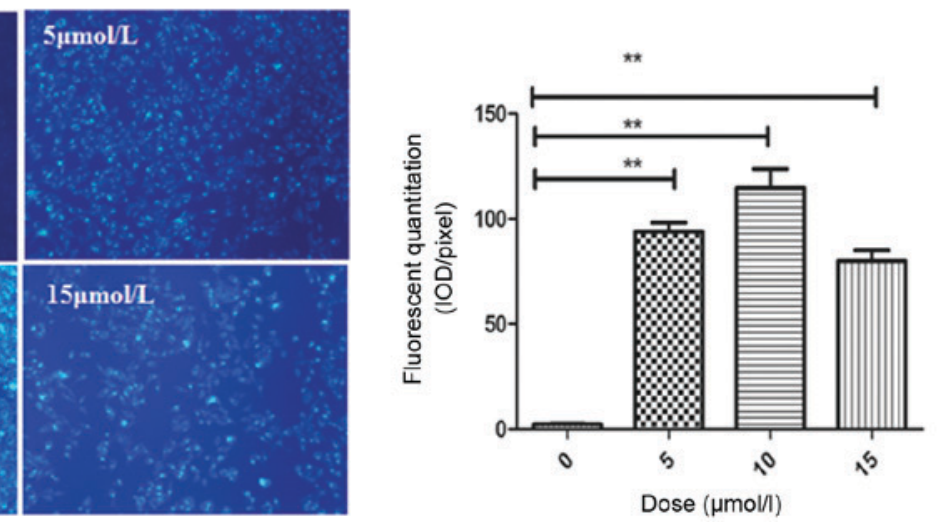

C

$\begin{array}{llll}0 & 5 & 10 & 15\end{array}$
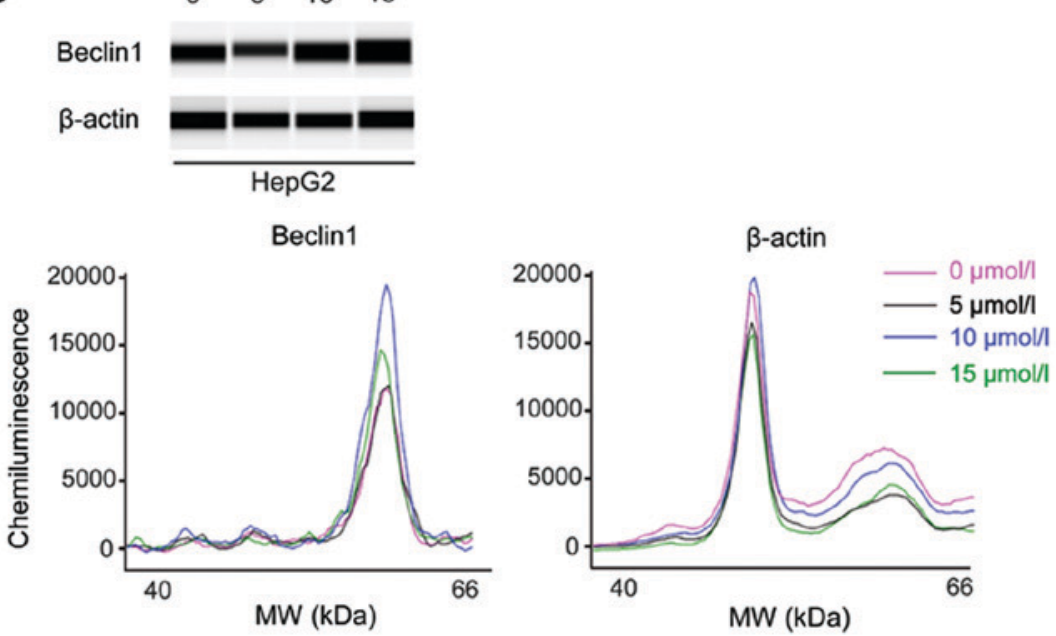

Figure 3. (A) Protein levels of Beclin1 and LC3-II/LC3-I were examined in 4 liver cancer cells treated with 0, 5, 10 and $15 \mu$ mol/1 rapamycin by western blot analysis. $\beta$-actin was used as an internal control. (B) Monodansylcadaverine method was used to detect the cell autophagy of HepG2 cells (magnification, $\mathrm{x} 400$ ). (C) The WES ${ }^{\mathrm{TM}}$ automated capillary-based size sorting system was used to measure the protein level of Beclin and $\beta$-actin in HepG2 cells. "P<0.05 and ${ }^{* *} \mathrm{P}<0.01$, as indicated. LC3, light chain 3; MW, molecular weight.

shown in four liver cancer cell lines under the treatment of $10 \mu \mathrm{mol} / 1$ rapamycin. These results were further confirmed in HepG2 cells by using MDC method and Protein Simple Western. The MDC-positive cells and the protein level of
Beclin1 were obviously increased in HepG2 cells after treated with rapamycin and peaked at $10 \mu \mathrm{mol} / 1$ (Fig. 3B and C). Taken together, these observations reveal that the autophagy could be triggered by rapamycin in four liver cancer cells. 
A

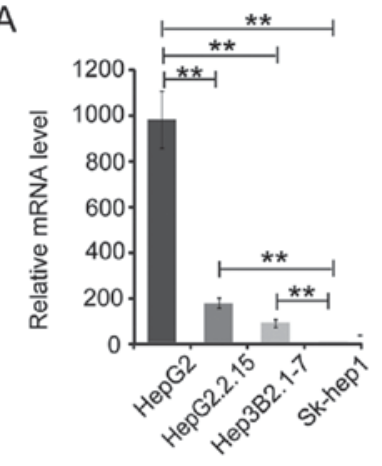

B
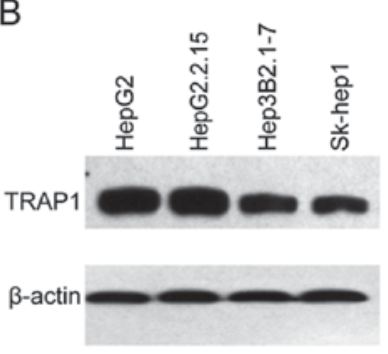
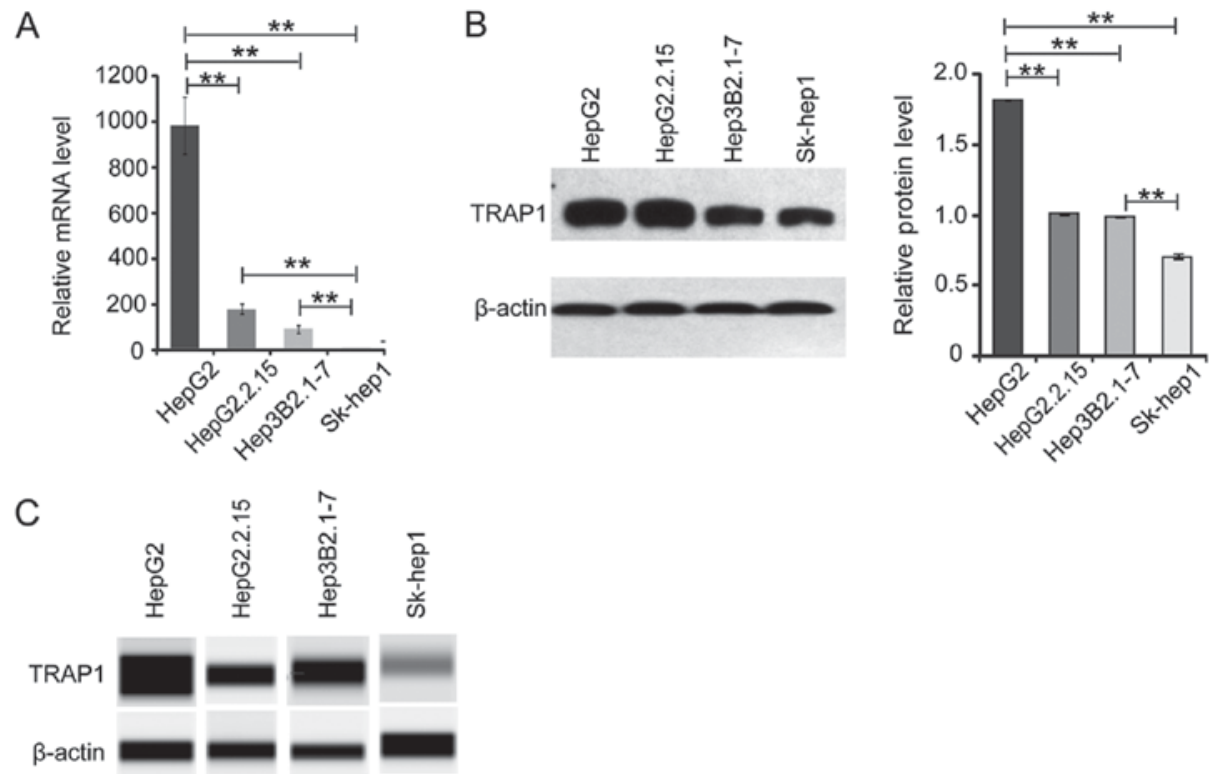

D

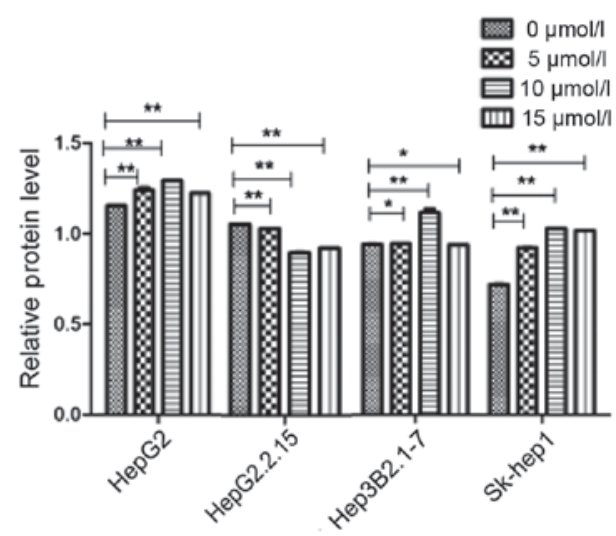

Figure 4. (A) Reverse transcription-quantitative polymerase chain reaction was used to detect the TRAP1 mRNA expression levels in Hep3b2.1-7, HepG2, HepG2.2.15 and Sk-hep1 cells. (B) The protein levels of TRAP1 in the 4 liver cancer cell lines were determined by western blot analysis. $\beta$-actin was used as an internal control. (C) WES ${ }^{\mathrm{TM}}$ automated capillary-based size sorting system was used to measure the protein level of TRAP1 and $\beta$-actin in 4 liver cancer cell lines. (D) The protein levels of TRAP1 were examined in 4 liver cancer cells treated with $0,5,10$ and $15 \mu$ mol/1 rapamycin by western blot analysis. $\beta$-actin was used as an internal control. " $\mathrm{P}<0.05$ and ${ }^{* * *} \mathrm{P}<0.01$, as indicated. TRAP1, tumor necrosis factor receptor-associated protein-1.

TRAP1 expression level was associated with cell autophagy. We further examined the mRNA and protein level of TRAP1 in four liver cancer cell lines. The expression level of TRAP1 was measured by RT-qPCR, western blot and Protein Simple Western. Intriguingly, a similar change of TRAP1 expression and autophagy activity was observed in four liver cancer cell lines with or without rapamycin induction. In detail, the expression level of TRAP1 was quite different in HepG2, Hep3b2.1-7 and Sk-hep1 cells, which representing different invasive ability. And the highest expression level of TRAP1 was observed in HepG2 cells which presented highest basal autophagy activity. Moreover, similar to autophagy activity, the TRAP1 expression was significantly inhibited in HepG2.2.15 cells (Fig. 4A-C). Furthermore, the TRAP1 expression level significantly enhanced in four rapamycin-treated liver cancer cell lines except HepG2.2.15 cells. And the TRAP1 expression peaked under the treatment of $10 \mu \mathrm{mol} / \mathrm{l}$ in HepG2, Hep3B2.1-7 and Sk-hep1 cells. Taken together, rapamycin might induce autophagy and TRAP1 expression in liver cancer cells, and the cell autophagy and TRAP1 expression might be positively correlated.

\section{Discussion}

In this study, we provided evidence that cell invasive ability, rapamycin and HBV infection were factors that affected the level of autophagy and TRAP1 expression in four liver cancer cell lines. Additionally, TRAP1 expression was positively correlated with the cell autophagy level, suggesting that TRAP1 and cell autophagy might be closely related.

Primary liver cancer patients have a high recurrence and mortality; however, they remain the main factors of poor prognosis (23). Liver cancer development is a multi-stage and multi-factor process, involving in multiple gene interactions and cell signaling pathways. Currently, specific clinical biomarkers are still not available, which has leaded to low early diagnosis. Increasing studies have identified the crucial role of autophagy in liver diseases (24). The dysregulation of autophagy is associated with viral hepatitis, non-alcoholic fatty liver disease, alcoholic liver disease, fibrosis, cirrhosis, and hepatocellular carcinoma (HCC) (25-28).

In the present study, we detected the cell growth of liver cancer cell lines with different invasive abilities, HBV 
infection and rapamycin exposure. The results showed that highly invasive Sk-hep1 cells grew faster than HepG2 cells and Hep3B2.1-7 cells. HepG2.2.15 cells, which were infected with HBV, grew slower than HepG2 cells. These results indicated that cell invasive ability might positively correlated with cell growth, and HBV infection might inhibit the growth of liver cancer cells. However, the potential mechanism remains to be determined. Rapamycin, a small-molecule material, is currently used to induce autophagy. We found that the growth rate of HepG2 cells was inhibited when exposed to 10 and $15 \mu \mathrm{mol} / 1$ rapamycin. The results demonstrated that high cell autophagy activity may inhibit cell growth. Some studies have shown that autophagy in cancer cells such as gastric cancer and liver cancer cells serves to 'maintain survival' and 'induce death', two opposing functions so autophagy could play a bidirectional role in those processes.

HBV infection is the most important cause of liver cancer (29). Some studies have found that HBV has a close relationship with cell autophagy (30-32). Moreover, TRAP1 expression is reported to associate with cell autophagy (33). To understand more about the pathogenesis of liver cancer, the expression level of Beclin1, LC3-II/LC3-Iand TRAP1 in four liver cancer cell lines was investigated by using RT-qPCR, western blot and Protein Simple Western. The results showed that the expression level of TRAP1, Beclin1 and LC3-II/LC3-I was highest in HepG2 cells and lowest in Sk-hep1 cells, suggesting that higher invasive ability and HBV infection could inhibit autophagy in liver cancer cell lines. Additionally, the expression level of TRAP1 showed a positive correlation with the autophagy level. The expression levels of Beclin1, LC3-II/LC3-I and TRAP1 was concentration-dependently increased in HepG2, Sk-hep1 and Hep3B2.1-7 cells after treated with 0,5 and $10 \mu \mathrm{mol} / 1$ rapamycin. However, the expression levels of Beclin1, LC3-II/LC3-I and TRAP1 were reduced when these cells were exposed to $15 \mu \mathrm{mol} / \mathrm{l}$ rapamycin. The expression level of TRAP1 was positively correlated with autophagy activity in HepG2, Sk-hep1 and Hep3B2.1-7 cells. Interestingly, the TRAP1 level in HepG2.2.15 cells exposed with 0,5 and $10 \mu \mathrm{mol} / 1$ rapamycin was negatively correlated with the rapamycin concentration, suggesting that the HBV infection can inhibit the rapamycin-induced TRAP1 expression. We found that the cell invasive ability, HBV infection and autophagy level can influence TRAP1 expression. However, the specific mechanism needs further study.

Cell autophagy is a biological process in eukaryotes with lysosome degradation. According to the cell environment, level of autophagy, and different roles of autophagy, it can be divided into basal autophagy and induced autophagy. Basal autophagy is a low-level cell autophagy activity that plays an important role in updating intracellular substances and maintaining the cell steady-state (34). Compared with basal autophagy, the reaction level of induced autophagy is higher, and the level of autophagy quickly climbs in a short time in the absence of external nutrients, an energy supply or other stimulation from the extracellular environment. An appropriate window of induced autophagy can maintain cell survival, but excessive induced autophagy can cause cell death (35). However, how autophagy switches from 'maintain survival' to 'induce death' is not fully clear. The present study also found that inducing autophagy was positively correlated with TRAP1 expression.
Thus, TRAP1 may be one of the triggers for the transformation. Additionally, it is thought that TRAP1 is an important factor related to cancer progression and prognosis. It can repair cell death with the properties of the matrix protein, Cyclophilin-D. Several preclinical studies, including in vivo studies in both normal and gene knock-out animal models, had confirmed that TRAP1 was a novel and efficient treatment target in various tumors. Although TRAP1 can maintain the mitochondrial integrity and function by inhibiting the fold of damaged proteins and promoting the refold process of denatured proteins, TRAP1 has dual effects on the regulation of mitochondrial apoptosis (36). Taken together, these results demonstrated that TRAP1 might play different roles in cell autophagy via the mitochondrial pathway. Thus, it is necessary to explore the factors that can affect TRAP1 expression and cell autophagy in liver cancer cell lines, and investigate the mechanism underlying the 'maintain survival' and 'induce death', which provide clues to improve the prognosis of liver cancer.

\section{Acknowledgements}

The authors would like to thank Miss Shan Wang (Department of Toxicology, Guangzhou Key Laboratory of Environmental Pollution and Health Risk Assessment, School of Public Health, Sun Yat-sen University, Guangzhou, P.R. China) for their assistance in revising the article.

\section{Funding}

The present study was supported financially by a grant from the National Natural Science Foundation of China (grant no. 81460446).

\section{Availability of data and materials}

The datasets used and/or analyzed during the current study are available from the corresponding author on reasonable request.

\section{Authors' contributions}

YS, $\mathrm{HZ}$ and $\mathrm{YL}$ were involved in the conception and design of the study, the acquisition and interpretation of data, drafting the article, and giving final approval of the version to be published. LY, MZ and XS contributed towards the design of the study. YY and WC analyzed and interpreted the data. YZ and JM acquired the data, and revised the article. All authors read and approved the final manuscript.

\section{Ethics approval and consent to participate}

Not applicable.

\section{Consent for publication}

Not applicable.

\section{Competing interests}

The authors declare that they have no competing interests. 


\section{References}

1. Kassebaum NJ, Bertozzi-Villa A, Coggeshall MS, Shackelford KA Steiner C, Heuton KR, Gonzalez-Medina D, Barber R, Huynh C, Dicker D, et al: Global, regional, and national levels and causes of maternal mortality during 1990-2013: A systematic analysis for the Global Burden of Disease Study 2013. Lancet 384: 980-1004, 2014.

2. Bosch FX and Ribes J: The epidemiology of primary liver cancer: Global epidemiology. Perspect Med Virol 6: 1-16, 2002.

3. Altekruse SF, McGlynn KA and Reichman ME: Hepatocellular carcinoma incidence, mortality, and survival trends in the United States From 1975 to 2005. J Clin Oncol 27: 1485-1491, 2009.

4. Sapisochin G, Goldaracena N, Laurence JM, Dib M, Barbas A, Ghanekar A, Cleary SP, Lilly L, Cattral MS, Marquez M, et al: The extended Toronto criteria for liver transplantation in patients with hepatocellular carcinoma: A prospective validation study. Hepatology 64: 2077-2088, 2016.

5. Cheng AL, Thongprasert S, Lim HY, Sukeepaisarnjaroen W, Yang TS, Wu CC, Chao Y, Chan SL, Kudo M, Ikeda M, et al Randomized, open-label phase 2 study comparing frontline dovitinib versus sorafenib in patients with advanced hepatocellular carcinoma. Hepatology 64: 774-784, 2016.

6. Zhu AX, Rosmorduc O, Evans TR, Ross PJ, Santoro A, Carrilho FJ, Bruix J, Qin S, Thuluvath PJ, Llovet JM, et al: SEARCH: A phase III, randomized, double-blind, placebo-controlled trial of sorafenib plus erlotinib in patients with advanced hepatocellular carcinoma. J Clin Oncol 33: 559-566, 2015.

7. Jing $\mathrm{K}$ and Lim $\mathrm{K}$ : Why is autophagy important in human diseases? Exp Mol Med 44: 69-72, 2012.

8. Wen X, Wu J, Wang F, Liu B, Huang C and Wei Y: Deconvoluting the role of reactive oxygen species and autophagy in human diseases. Free Radic Biol Med 65: 402-410, 2013.

9. Yang Z and Klionsky DJ: Eaten alive: A history of macroautophagy. Nat Cell Biol 12: 814-822, 2010.

10. Mizushima N: Autophagy: Process and function. Genes Dev 21: 2861-2873, 2007.

11. Klionsky DJ, Cregg JM, Dunn WA Jr, Emr SD, Sakai Y, Sandoval IV, Sibirny A, Subramani S, Thumm M, Veenhuis M and Ohsumi Y: A unified nomenclature for yeast utophagy-related genes. Dev Cell 5: 539-545, 2003.

12. Mathew R and White E: Why sick cells produce tumors: The protective role of autophagy. Autophagy 3: 502-505, 2007.

13. White E: Deconvoluting the context-dependent role for autophagy in cancer. Nat Rev Cancer 12: 401-410, 2012.

14. Kenerson HL, Yeh MM, Kazami M, Jiang X, Riehle KJ, McIntyre RL, Park JO, Kwon S, Campbell JS and Yeung RS: Akt and mTORC1 have different roles during liver tumorigenesis in mice. Gastroenterology 144: 1055-1065, 2013.

15. Zeng H, Yang K, Cloer C, Neale G, Vogel P and Chi H: mTORC1 couples immune signals and metabolic programming to establish T(reg)-cell function. Nature 499: 485-490, 2013.

16. Costantino E, Maddalena F, Calise S, Piscazzi A, Tirino V, Fersini A, Ambrosi A, Neri V, Esposito F and Landriscina M: TRAP1, a novel mitochondrial chaperone responsible for multi-drug resistance and protection from apoptotis in human colorectal carcinoma cells. Cancer Lett 279: 39-46, 2009.

17. Choi H, Merceron C, Mangiavini L, Seifert EL, Schipani E, Shapiro IM and Risbud MV: Hypoxia promotes noncanonical autophagy in nucleus pulposus cells independent of MTOR and HIF1A signaling. Autophagy 12: 1631-1646, 2016.

18. Caino MC, Chae YC, Vaira V, Ferrero S, Nosotti M, Martin NM, Weeraratna A, O'Connell M, Jernigan D, Fatatis A, et al: Metabolic stress regulates cytoskeletal dynamics and metastasis of cancer cells. J Clin Invest 123: 2907-2920, 2013.

19. Siegelin MD: Inhibition of the mitochondrial Hsp90 chaperone network: A novel, efficient treatment strategy for cancer? Cancer Lett 333: 133-146, 2013.
20. Costa AC, Loh SH and Martins LM: Drosophila Trap1 protects against mitochondrial dysfunction in a PINK1/parkin model of Parkinson's disease. Cell Death Dis 4: e467, 2013.

21. Chen JF, Wu QS, Xie YX, Si BL, Yang PP, Wang WY, Hua Q and He Q: TRAP1 ameliorates renal tubulointerstitial fibrosis in mice with unilateral ureteral obstruction by protecting renal tubular epithelial cell mitochondria. FASEB J 31: 4503-4514, 2017.

22. Harris VM: Protein detection by Simple Western ${ }^{\mathrm{TM}}$ analysis. Methods Mol Biol 1312: 465-468, 2015.

23. Xiao S, Chang RM, Yang MY, Lei X, Liu X, Gao WB, Xiao JL and Yang LY: Actin-like 6A predicts poor prognosis of hepatocellular carcinoma and promotes metastasis and epithelial-mesenchymal transition. Hepatology 63: 1256-1271, 2016.

24. Huang Q, Zhan L, Cao H, Li J, Lyu Y, Guo X, Zhang J, Ji L, Ren T, An J, et al: Increased mitochondrial fission promotes autophagy and hepatocellular carcinoma cell survival through the ROS-modulated coordinated regulation of the NFKB and TP53 pathways. Autophagy 12: 999-1014, 2016.

25. Wang L, Liu X, Nie J, Zhang J, Kimball SR, Zhang H, Zhang WJ, Jefferson LS, Cheng Z, Ji Q and Shi Y: ALCAT1 controls mitochondrial etiology of fatty liver diseases, linking defective mitophagy to steatosis. Hepatology 61: 486-496, 2015.

26. Ding WX, Li M and Yin XM: Selective taste of ethanol-induced autophagy for mitochondria and lipid droplets. Autophagy 7: 248-249, 2011.

27. Lodder J, Denaës T, Chobert MN, Wan J, El-Benna J, Pawlotsky JM, Lotersztajn S and Teixeira-Clerc F: Macrophage autophagy protects against liver fibrosis in mice. Autophagy 11: 1280-1292, 2015.

28. Ueno T and Komatsu M: Autophagy in the liver: Functions in health and disease. Nat Rev Gastroenterol Hepatol 14: 170-184, 2017.

29. Samal J, Kandpal M and Vivekanandan P: Molecular mechanisms underlying occult hepatitis B virus infection. Clin Microbiol Rev 25: 142-163, 2012.

30. Shin GC, Kang HS, Lee AR and Kim KH: Hepatitis B virus-triggered autophagy targets TNFRSF10B/death receptor 5 for degradation to limit TNFSF10/TRAIL response. Autophagy 12: 2451-2466, 2016.

31. Lan SH, Wu SY, Zuchini R, Lin XZ, Su IJ, Tsai TF, Lin YJ, Wu CT and Liu HS: Autophagy suppresses tumorigenesis of hepatitis B virus-associated hepatocellular carcinoma through degradation of microRNA-224. Hepatology 59: 505-517, 2014.

32. Sir D, Ann DK and Ou JH: Autophagy by hepatitis B virus and for hepatitis B virus. Autophagy 6: 548-549, 2010.

33. Altieri DC: Mitochondrial HSP90s and tumor cell metabolism. Autophagy 9: 244-245, 2013.

34. Guo XL, Hu F, Zhang SS, Zhao QD, Zong C, Ye F, Guo SW, Zhang JW, Li R, Wu MC and Wei LX: Inhibition of p53 increases chemosensitivity to 5-FU in nutrient-deprived hepatocarcinoma cells by suppressing autophagy. Cancer Lett 346: 278-284, 2014.

35. Amaravadi R, Kimmelman AC and White E: Recent insights into the function of autophagy in cancer. Genes Dev 30: 1913-1930, 2016.

36. Pridgeon JW, Olzmann JA, Chin LS and Li L: PINK1 protects against oxidative stress by phosphorylating mitochondrial chaperone TRAP1. PLoS Biol 5: e172, 2007.

(7) $($ This work is licensed under a Creative Commons Attribution-NonCommercial-NoDerivatives 4.0 International (CC BY-NC-ND 4.0) License. 